\title{
Synthesis and characterization of poly (Itaconic Acid-m-Phen- ylenediamine)
}

\author{
Chuanxing Wang, Pengfei Wang, Qinhong $\mathrm{Xu}$, Zhe Wei and Yumin $\mathrm{Wu}^{\mathrm{a}}$ \\ College of Chemical Engineering, Qingdao University of Science \& Technology, Qingdao 266042, China
}

\begin{abstract}
With Itaconyl chloride and m-phenylenediamine as raw materials, poly(itaconic acid- m-phenylenediamine) was synthesized by the low temperature solution polycondensation we reported. The effects of initial temperature and reaction time on polycondensation were investigated. And the product was characterized by ${ }^{1} \mathrm{H}-\mathrm{NMR}$, FT-IR and TGA. The results showed that the polycondensation should carry out at a low temperature. The initial temperature should be lower $0{ }^{\circ} \mathrm{C}$. The polycondensation time was $90-100$ minutes. Under these conditions, the thermal stability was good and its inherent viscosity was about 1.4. The spectra of FT-IR and ${ }^{1} \mathrm{H}-\mathrm{NMR}$ confirmed its structure.
\end{abstract}

Keywords: low temperature solution polycondensation; itaconyl chloride; polymer; inherent viscosity; characterization.

\section{Introduction}

Polyamide (PA) is a widely used engineering plastic. It has excellent physical and mechanical properties because of its special structure and the role of the crystal [1,2]. The most important PAs are Polyamide 6fibers and Polyamide 66 fibers. They are essential products of daily life and industrial circle [3].

There are various methods of PA preparation, such as low temperature solution polycondensation[4], gaseous polymerization[5], interfacial polymerization[6] and emulsion polymerization. The usage of solvent is the general feature of low temperature solution polycondensation. With the solvent, the polymerization could proceed at low reaction temperature and mild condition. Moreover, there are several advantages, such as good for heat exchange and solution of indissolvable solutes. Low temperature solution polycondensation was used widely because of low consumption of solvent, high efficiency. The product, resin solution, could be used directly to spinning, pulping or filming without precipitation, water washing and dissolving of resin. So, it was more economical [7]. Interfacial polycondensation was a method that two monomers dissolved in the water and solvent not miscible with water, respectively. The reaction was carried out at the water and oil interface. It has several advantages, such as simple devices, high reaction speed and the product with high molecule. Polyamide, polyarylester, makrolon and polyurethane were prepared usually by this method. Otherwise, the consumption of solvent was very large, and the efficiency of devices was low $[8,9]$. With emulsion polymerization, the product was the emulsion with insoluble polymer dispersed in continuous phase. By the emulsion polymerization, the insoluble polymer was dispersed

a Correspondence author : wuyumin005@163.com 
stably in the continuous phase. And, the main chain could be modified by altering feeding sequence with comonomer[10].

Poly (itaconic acid-m-phenylenediamine), as a new resin material, was reported in few articles. As an organic plasticizer for poly (butylene succinate) (PBS)[11] and polyvinyl chloride (PVC)[12], it has a good plasticizing effect. In this paper, poly (itaconic acid-m-phenylenediamine) was prepared by low temperature solution polycondensation. The reaction mechanism and condition were analyzed. And its structure was characterized.

\section{Experimental}

\subsection{Materials}

Itaconic acid (industrial product) was purchased from Qingdao Langyatai Group Co., China. Dimethylacetamide(analytical reagent, AR), m-phenylenediamine(AR) and $\mathrm{Ca}(\mathrm{OH})_{2}(\mathrm{AR})$ was purchased from Sinopharm chemical reagent Co., Ltd..

\subsection{Synthesis of polyamide}

Anhydrous m-phenylenediamine was dissolved with moderate dimethylacetamide in the reactor. Itaconyl chloride was dropped into the reactor with agitating at $0{ }^{\circ} \mathrm{C}$. Then, the reagents were heated to reactive temperature. When the reaction was completed, $\mathrm{Ca}(\mathrm{OH})_{2}$ powder was added and kept agitating for 30 minutes. The crude product was obtained. And then, impurities were filtered out by distilled water. Water was removaled by vacuum drying oven, and gave the pure product.

\subsection{Characterization}

${ }^{1}$ H-NMR Spectroscopy: Chemical structures of PA were characterized by ${ }^{1} \mathrm{H}-\mathrm{NMR}$, which was implemented on a BRUKER AV $500 \mathrm{MHz}$ spectrometer at ambient temperature, using dimethyl sulfoxide (DMSO) as the solvent and the internal standard, respectively.

FT-IR: Fourier Transform Infrared Spectrometer was carried out with a BRUKER TENSOR-27 infrared spectrometer using $\mathrm{KBr}$ pellets to confirm the structure.

Thermal properties: Thermo gravimetric Analysis: The thermal stabilities of polymers were determined through Thermo gravimetric Analysis (TGA). The thermograms were recorded on a STA409PCLuxx thermo gravimetric analyzer from room temperature to $600{ }^{\circ} \mathrm{C}$ at a heating rate of 15 ${ }^{\circ} \mathrm{C} /$ min under $\mathrm{N}_{2}$ atmosphere.

\section{Results and discussion}

\subsection{Effects of reaction temperature}

The polymerization was very strong because of high reactivity of both monomers. So, several side reactions would occur. The effects of initial temperature were discussed to control the side reactions to do not affect the main reaction.

Figure 1 showed the effects of initial temperature on the inherent viscosity. When the initial temperature was lower than $0{ }^{\circ} \mathrm{C}$, the inherent viscosity was about 1.4 . When the initial temperature increased, the inherent viscosity of the product decreased sharply according to Figure 1 . The inherent viscosity was similar when the initial temperature was $-5{ }^{\circ} \mathrm{C}$ and $0{ }^{\circ} \mathrm{C}$. But the energy consumption with the initial temperature $-5^{\circ} \mathrm{C}$ was greater than that of $0{ }^{\circ} \mathrm{C}$. So, $0^{\circ} \mathrm{C}$ was the best initial temperature. 


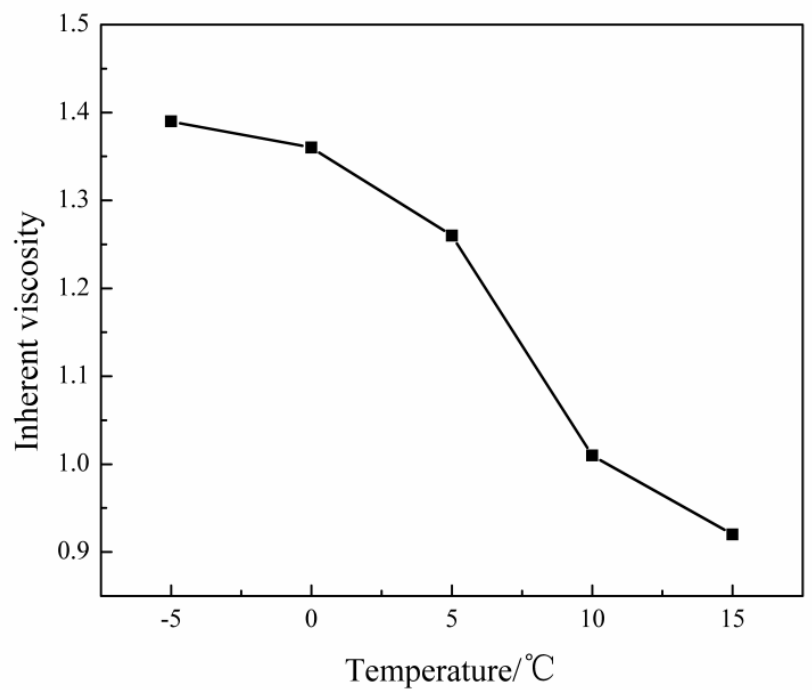

Figure 1. The relationship of initial reaction temperature and the inherent viscosity

\subsection{Effects of reaction temperature}

The effects of reaction temperature and reaction time on the inherent viscosity were studied at the same initial temperature. During the reaction, the sample was taken out to measure the inherent viscosity every 20 minutes. The date was showed in Figure 2.

According to Figure 2, inherent viscosity of the product was high at low temperature. With the increasing of reaction temperature, inherent viscosity decreased constantly. The inherent viscosity was highest at $0{ }^{\circ} \mathrm{C}$. So, it was the best condition.

The inherent viscosity increased sharply in the first 20 minutes. Then, it changed slowly in the 50-90 minutes. After 90 minutes, it was beginning to stabilize. At the beginning of the reaction, reagents' concentration was high. So, the reaction speed was high. As the reaction was carried out, the materials were consumed and the concentration decreased. So, the reaction speed slowed down. When the most materials were consumed after about 90 minutes, the reaction ceased. Therefore, the suitable reaction time was 90-100 minutes.

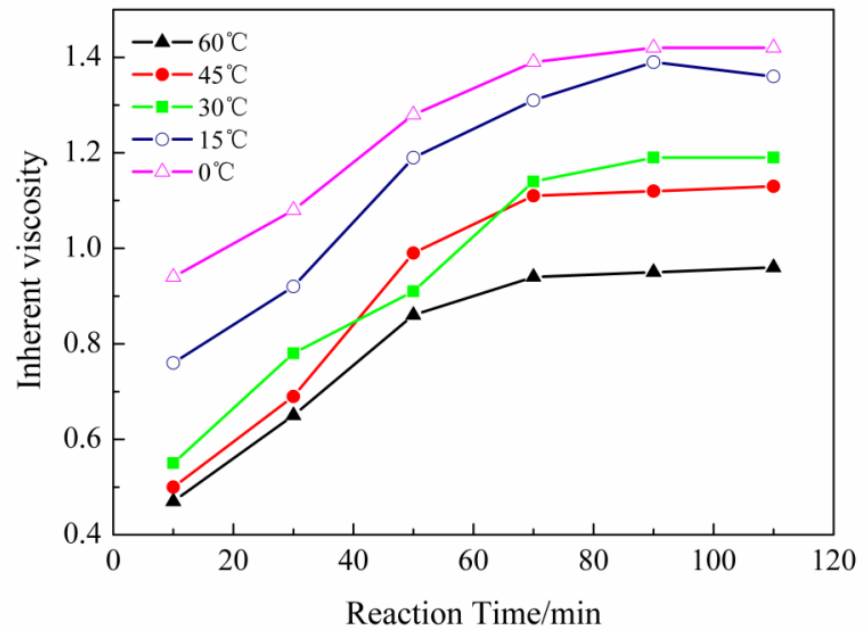

Figure 2. The relationship of reaction time and inherent viscosity 


\section{$3.3^{1} \mathrm{H}-\mathrm{NMR}$ of product}

The structure of poly (itaconic acid-m-phenylenediamine) was below:

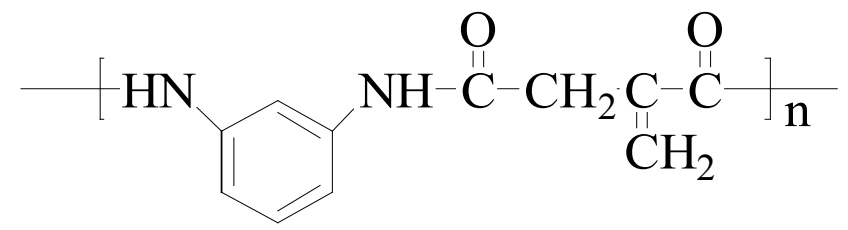

Figure 3. The structure of poly (itaconic acid-m-phenylenediamine)

${ }^{1} \mathrm{H}-\mathrm{NMR}$ spectrum of the product was showed in Figure 4. In the 1H-NMR spectra, the peak around $2.45 \mathrm{ppm}$ was assigned to the underlined protons in $-\mathrm{CH}_{2}-$. The peaks around 5.96 and 5.61 ppm were attributed to the protons signal of the group $-\mathrm{C}=\mathrm{CH}_{2}$. The ratio of these peaks area was $1: 1$. The continuous peaks from 6.22 to 7.18 were attributed to the protons of benzene ring. The continuous peaks around 4.07 and 7.18 were attributed to the proton of -NH-. The most intense peak around 3.27 was to residual water.

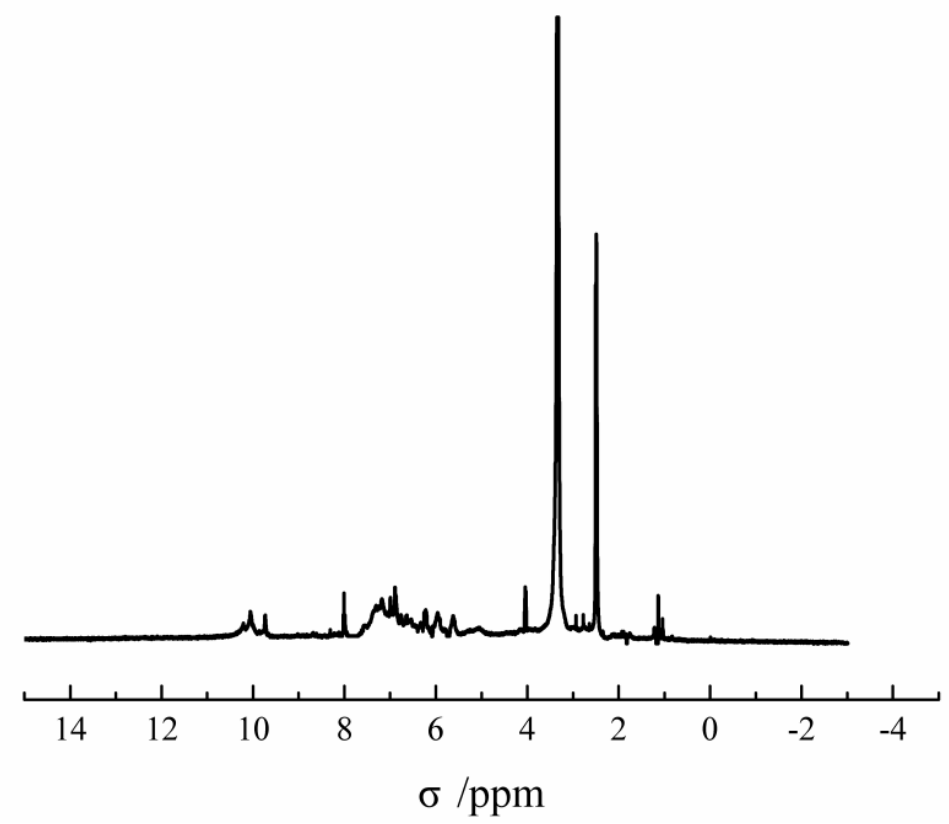

Figure 4. ${ }^{1} \mathrm{H}-\mathrm{NMR}$ spectrum of the product 


\subsection{FT-IR spectrum of product}

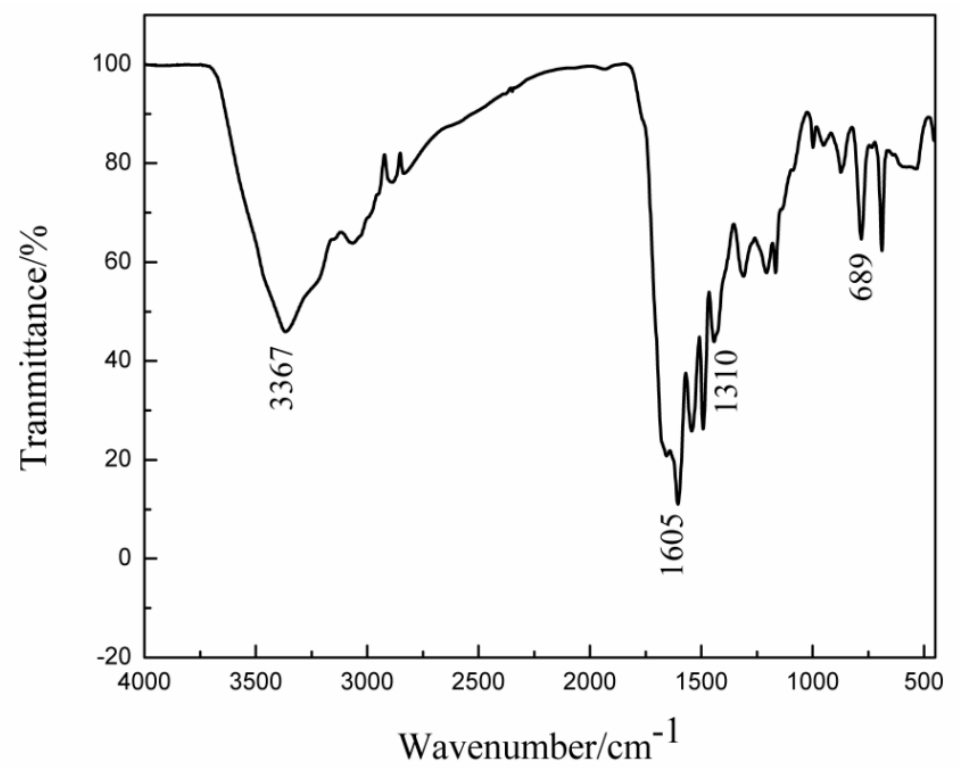

Figure 5. FT-IR spectrum of product

The FT-IR spectrum of poly(itaconic acid-m-phenylenediamine) was shown in Figure 5. In the spectrum of the polymer, the intense absorption at $3367 \mathrm{~cm}^{-1}$ was assigned to the stretching vibration of N-H in the amide group. Four absorption peaks from $1605 \mathrm{~cm}^{-1}$ to $1442 \mathrm{~cm}^{-1}$ was attributed to the amide group. Three small peaks from $1166 \mathrm{~cm}^{-1}$ to $1310 \mathrm{~cm}^{-1}$ was assigned to the stretching vibration of C-N in amide group and in-place bending vibration of N-H. Five peaks from $689 \mathrm{~cm}^{-1}$ to $1002 \mathrm{~cm}^{-1}$ were to in-place bending vibration of $\mathrm{C}-\mathrm{H}$ in aromatic ring.

\subsection{TG and DTG}

Thermogravimetric analysis could confirm the decomposition temperature, decomposition speed and weight loss ratio of the polymer. Then, its thermostability could be evaluated. In this article, thermogravimetric analysis was made at $\mathrm{N}_{2}$ atmosphere, $50 \mathrm{~mL} / \mathrm{min}$ of gas stream and $15{ }^{\circ} \mathrm{C} / \mathrm{min}$ of heating rate. TG and DTG curves were shown in Figure 6.

From Figure 6 , the beginning weight loss was due to the water in the product. When the temperature was above $245^{\circ} \mathrm{C}$, poly (itaconic acid-m-phenylenediamine) decomposed slowly. When the temperature was to about $300{ }^{\circ} \mathrm{C}$, the decomposition was fast. The peak of decomposition was $-20.37 \% / \mathrm{min}$ at $370{ }^{\circ} \mathrm{C}$. The final weight loss was $-96.54 \%$. 


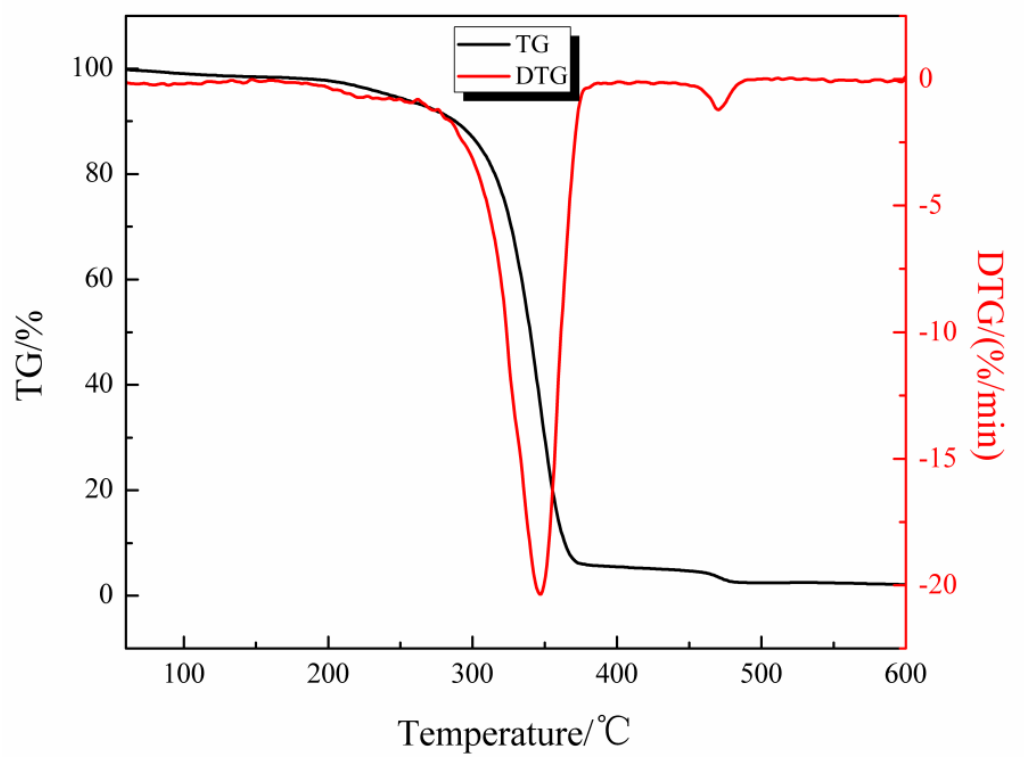

Figure 6. TG and DTG curves of product

\section{Conclusions}

With itaconyl chloride and m-phenylenediamine as raw materials, poly(itaconic acid-m-phenylenediamine) was prepared by low temperature solution polycondensation. The reaction conditions were studied. And the structure and thermal properties were characterized. The results showed that the polycondensation should carry out at a low temperature. The initial temperature should be lower $0{ }^{\circ} \mathrm{C}$. The polycondensation time was 90-100 minutes. Under these conditions, the thermal stability was good and its inherent viscosity was about 1.4. The spectra of FT-IR and ${ }^{1} \mathrm{H}-\mathrm{NMR}$ confirmed its structure.

\section{Acknowledgement}

The contract grant sponsor is National Natural Science Foundation of China (21306094).

\section{References}

1. L. L. Zhang, P. Fu, Z. Cui, M.Y. Liu, Q.F. Zhao. Progress in synthesis of optically active polyamide Engineering [J]. Plastics Application, 43, 133-136 (2015)

2. F. Qiao,Q. Li, Z. N. Qi, F. S. Wang. Synthesis and Characterization of Nylon 6/Clay Nanocomposites [J]. Polymer Bulletin, 3, 135-143 (1997)

3. C. Song, M. J. Wen, Y. Yu. Status and outlook of polyamide fiber production [J]. China synthetic fiber industry, 35, 49-53 (2012)

4. Z. W. Liu. Study on the polymerization and spinning of poly(m-phenyleneisophthalamide) [D]. Shanhai, Donghua University, 2004

5. L. Chen, Z. W. Liu, W. L. Pan, Z. F. Liu. Study on Preparation of Poly (m-phenyleneisophthalamide) by the Method of Low-temperature Solution Polycondensation[J]. Journal of Donghua University(Natural Science), 30, 132-134 (2004)

6. J. Geng. Study on the Polymerization Technique of Poly(m-phenylene terephthalamide)(aramid 1313) [D]. Jilin, Changchun University of Techology, 2012

7. G. H. Ma, Z. G. Su. New polymer material [M]. Beijing, Chemical industry press, 3, 406-417 
(2003)

8. S. Z. Wang, Q. R. Wang, Z. F. Liu.Introduction to high tech fiber [M]. Shanghei, China textile university press, 338-347 (1999)

9. H. W. Hill, K. S. Louise, M. P. Winthrop. Polyamides from reaction of aromatic diacid halide dissolved in cyclic nonaromatic oxygenated organic solvent and an aromatic diamine[P]: US, US 3006899 A, 1961.

10. S. Ozawa, F. Hiroshi. PROCESS FOR PREPARATION OF POLYAMIDES[P]: US, US3640970, 1969

11. Z. C. Liang, P. J. Pan, B. Zhu, et al. Mechanical and thermal properties of poly(butylene succinate)/plant fiber biodegradable composite[J]. Journal of Applied Polymer Science, 115, 3559-3567 (2010)

12. P. Jia, C. Bo, L. Hu, et al. Synthesis of a novel polyester plasticizer based on glyceryl monooleate and its application in poly(vinyl chloride)[J]. Journal of Vinyl \& Additive Technology, DOI: $10.1002 /$ vnl.21468, early view 2015 